TITLE:

\title{
Molecular-packing-enhanced charge transport in organic field- effect transistors based on semiconducting porphyrin crystals
}

$\operatorname{AUTHOR}(S)$ :

Minari, T; Seto, M; Nemoto, T; Isoda, S; Tsukagoshi, $\mathrm{K}$; Aoyagi, Y

\section{CITATION:}

Minari, T ... [et al]. Molecular-packing-enhanced charge transport in organic field-effect transistors based on semiconducting porphyrin crystals. APPLIED PHYSICS LETTERS 2007, 91(12): 123501.

ISSUE DATE:

2007-09-17

URL:

http://hdl.handle.net/2433/50343

\section{RIGHT:}

Copyright 2007 American Institute of Physics. This article may be downloaded for personal use only. Any other use requires prior permission of the author and the American Institute of Physics. 


\title{
Molecular-packing-enhanced charge transport in organic field-effect transistors based on semiconducting porphyrin crystals
}

\author{
Takeo Minaria) and Mari Seto \\ Institute for Chemical Research, Kyoto University, Uji, Kyoto 611-0011, Japan and RIKEN, 2-1 Hirosawa, \\ Wako, Saitama 351-0198, Japan
}

Takashi Nemoto and Seiji Isoda

Institute for Chemical Research, Kyoto University, Uji, Kyoto 611-0011, Japan

Kazuhito Tsukagoshi and Yoshinobu Aoyagi

RIKEN, 2-1 Hirosawa, Wako, Saitama 351-0198, Japan and CREST, JST, 4-1-8 Honcho, Kawaguchi, Saitama 332-0012, Japan

(Received 18 July 2007; accepted 28 August 2007; published online 18 September 2007)

\begin{abstract}
The effect of semiconductor crystal structure on the performance of organic field-effect transistors is investigated through the fabrication and characterization of devices based on single porphyrin crystals with different center metals. The field-effect mobility of the transistors is found to increase with decreasing intermolecular distance, attributable to greater overlap of $\pi$ orbitals among close-packed molecules and the consequent promotion of charge transport. (C) 2007 American Institute of Physics. [DOI: 10.1063/1.2786020]
\end{abstract}

Charge carrier mobility in single crystals and thin films of organic conjugated materials has been investigated in attempts to improve electrical transport in organic semiconductors and thereby enhance the performance of organic electronic devices. ${ }^{1}$ Extensive research on electrical conduction in organic field-effect transistors ${ }^{2-6}$ (OFETs) has revealed that the field-effect mobility $\left(\mu_{\mathrm{FET}}\right)$ is dependent not only on the fundamental properties of the molecules but also on factors such as grain size, interfacial modifications, and semiconductor thickness. ${ }^{7-10}$ The crystal structure of organic semiconductors is expected to have a considerable influence on OFET performance because larger overlap of $\pi$ orbitals among neighboring molecules may increase the bandwidth and facilitate the charge transport. ${ }^{11-13}$ The relationship between crystal structure and $\mu_{\mathrm{FET}}$, however, has not been confirmed yet due to the masking of such intrinsic conduction properties by the effects of structural defects and disorder at grain boundaries in thin films. To investigate the effect of crystal structure, it is therefore desirable to fabricate OFETs from single crystals or grains. ${ }^{5,6,14-18}$ Rubrene and pentacene single-crystal transistors, for example, have recently been shown to exhibit anisotropic charge transport. ${ }^{19,20}$

Porphyrin is an important complex molecule that offers a variety of optical, electrochemical, and catalytic properties dependent on the center metal and chemical modifications. In comparison with compounds such as acene, however, OFETs using metal-complex molecules have attracted little attention despite their being good candidates as electronic materials. ${ }^{21-23}$ In this letter, we fabricated and characterized field-effect transistors based on single porphyrin crystals. Different center metals of porphyrin molecules offers a variety of intermolecular distances within the crystal, which revealed a clear relationship between the $\mu_{\mathrm{FET}}$ and crystal structure.

Metallo-porphyrin $\quad(2,3,7,8,12,13,17,18$-octaethyl-21 $H$, $23 \mathrm{H}$-metallo-porphyrin) (M-OEP) crystals with various cen-

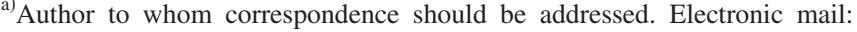
minari@ riken.jp
}

ter metals were grown by recrystallization from chloroform/ hexane solution. Saturated chloroform solutions of M-OEP molecules were prepared, and hexane was added to make the vaporizing rate of chloroform slower. As chloroform was naturally vaporized, the M-OEP molecules crystallized out of the solution. The crystals were obtained as bundles of needlelike twin crystals which are single crystalline in the longitudinal direction. Each crystal was several tens of micrometers in width and several micrometers in thickness. The crystal structures of the M-OEP crystals were determined by X-ray diffraction (XRD) analysis using a Rigaku R-AXIS RAPID diffractometer with Mo $K \alpha$ radiation at room temperature.

Four different metals, cobalt $(\mathrm{Co})$, copper $(\mathrm{Cu})$, zinc $(\mathrm{Zn})$, and palladium $(\mathrm{Pd})$, were tested as porphyrin center atoms [Fig. 1(a)]. The crystal structures obtained for Co-, $\mathrm{Cu}-$, and $\mathrm{Zn}$-OEP in the present study are similar with those

(a)

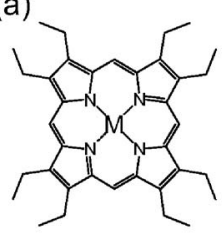

(b)

$\mathrm{M}: \mathrm{Co}, \mathrm{Cu}, \mathrm{Zn}, \mathrm{Pd}$
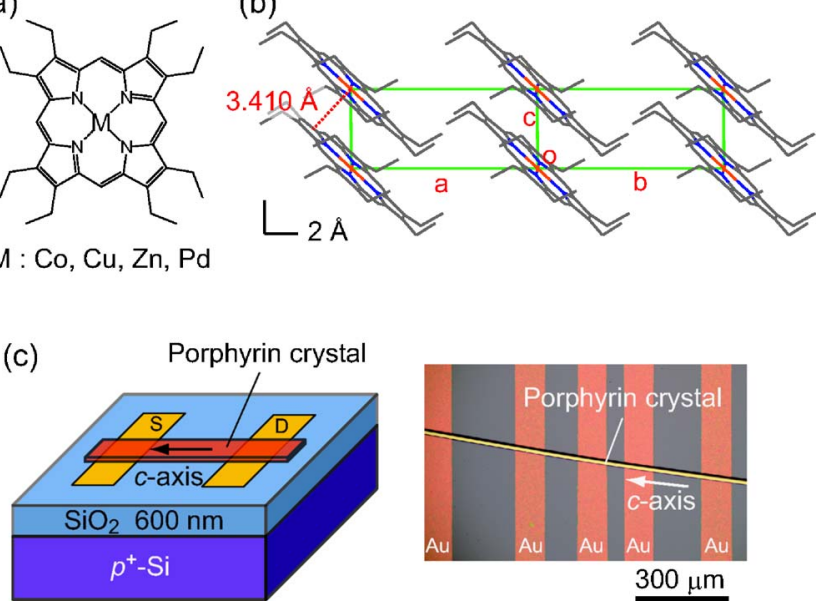

FIG. 1. (Color online) (a) Molecular structure of M-OEPs. (b) Crystal structure of Pd-OEP projected along the 110 axis with intermolecular distance $(3.410 \AA$ ) indicated by red dashed line. (c) Schematic structure and typical optical micrograph of porphyrin-crystal-based field-effect transistor. The longitudinal direction of the needlelike crystal corresponds to the $c$ axis 
TABLE I. Crystallographic parameters and field-effect mobility of M-OEP crystals. $D$ indicates intermolecular distance among neighboring molecules.

\begin{tabular}{lcccccccc}
\hline \hline Crystal & $a(\AA)$ & $b(\AA)$ & $c(\AA)$ & $\alpha\left(^{\circ}\right)$ & $\beta\left(^{\circ}\right)$ & $\gamma\left({ }^{\circ}\right)$ & $D(\AA)$ & $\begin{array}{c}\mu_{\text {FET }} \\
\left(\mathrm{cm}^{2} / \mathrm{V} \mathrm{s}\right)\end{array}$ \\
\hline Co-OEP & 12.922 & 13.033 & 4.766 & 92.130 & 92.785 & 113.156 & 3.338 & 0.200 \\
Cu-OEP & 13.263 & 13.274 & 4.785 & 92.841 & 92.927 & 113.227 & 3.386 & 0.068 \\
Zn-OEP & 13.342 & 13.259 & 4.770 & 91.488 & 93.249 & 113.128 & 3.393 & 0.036 \\
Pd-OEP & 13.313 & 13.325 & 4.776 & 93.632 & 92.520 & 113.301 & 3.410 & 0.014 \\
\hline \hline
\end{tabular}

in previous reports. ${ }^{24-26}$ No structural data have been reported so far for the Pd-OEP crystal. The present XRD analyses revealed that these four M-OEP crystals have similar molecular arrangements, all belonging to space group $P-1$ and having the same triclinic crystalline lattice with slightly different lattice parameters (Table I). This allowed us to investigate the conduction of organic crystals which have similar molecular arrangements and differing intermolecular distances. The lattice parameters determined for Pd-OEP crystal are $a=13.313 \AA, b=13.325 \AA, c=4.776 \AA, \alpha=93.632^{\circ}, \beta$ $=92.520^{\circ}$, and $\gamma=113.301^{\circ}$, and the crystal structure is shown in Fig. 1(b). The porphyrin molecules are situated in face-to-face relationships along the $c$ axis with large overlap among $\pi$ orbitals, which is expected to contribute to charge transport. Since the $c$ axis corresponds to longitudinal direction of the needlelike crystals, the electrical conduction was measured along the $c$ axis [Fig. 1(c)].

Field-effect devices were fabricated by placing the crystals onto the substrates on which source and drain electrodes were prefabricated. ${ }^{16,17}$ Substrates were highly doped $p^{+}$silicon wafers (gate electrode) with a 600-nm-thick thermally grown oxide layer (gate insulator). Source and drain electrodes were fabricated on the substrates by thermal evaporation of titanium $(3 \mathrm{~nm})$ and gold $(17 \mathrm{~nm})$, respectively. A hydrophobic silicon dioxide surface was obtained by treatment with octadecyltrichlorosilane. Then, the flexible
M-OEP crystal was placed onto the electrodes [Fig. 1(c)]. The crystal strongly adhered to silicon dioxide surface and made good electrical contact with the electrodes.

Electrical measurements were carried out under vacuum $\left(10^{-3} \mathrm{~Pa}\right)$ conditions in a dark environment. The devices were operated in $p$-channel accumulation mode. Typical current-voltage characteristics for the four porphyrin FETs are shown in Fig. 2. All four crystals exhibited good fieldeffect characteristics with well-defined saturation regions. The linear increase in drain current in the low gate voltage region indicates Ohmic contact between the source/drain electrodes and the organic crystals. The field-effect mobility was calculated from the drain current in the saturation region $\left(I_{D, \text { sat }}\right)$ by

$$
I_{D, \mathrm{sat}}=\frac{\mu_{\mathrm{FET}} W C_{i}}{2 L}\left(V_{G}-V_{T}\right)^{2},
$$

where $L$ and $W$ are the channel length and width, $C_{i}$ is the insulator capacitance per unit area, and $V_{G}$ and $V_{T}$ are the gate and threshold voltages. The channel width is determined by the width of the crystals, which was estimated from polarized optical micrograph. The results for the four porphyrin devices are summarized in Table I. The obtained values of $\mu_{\mathrm{FET}}$ can be seen to be dependent on the center metal. The Co-OEP crystal exhibited the highest mobility among the
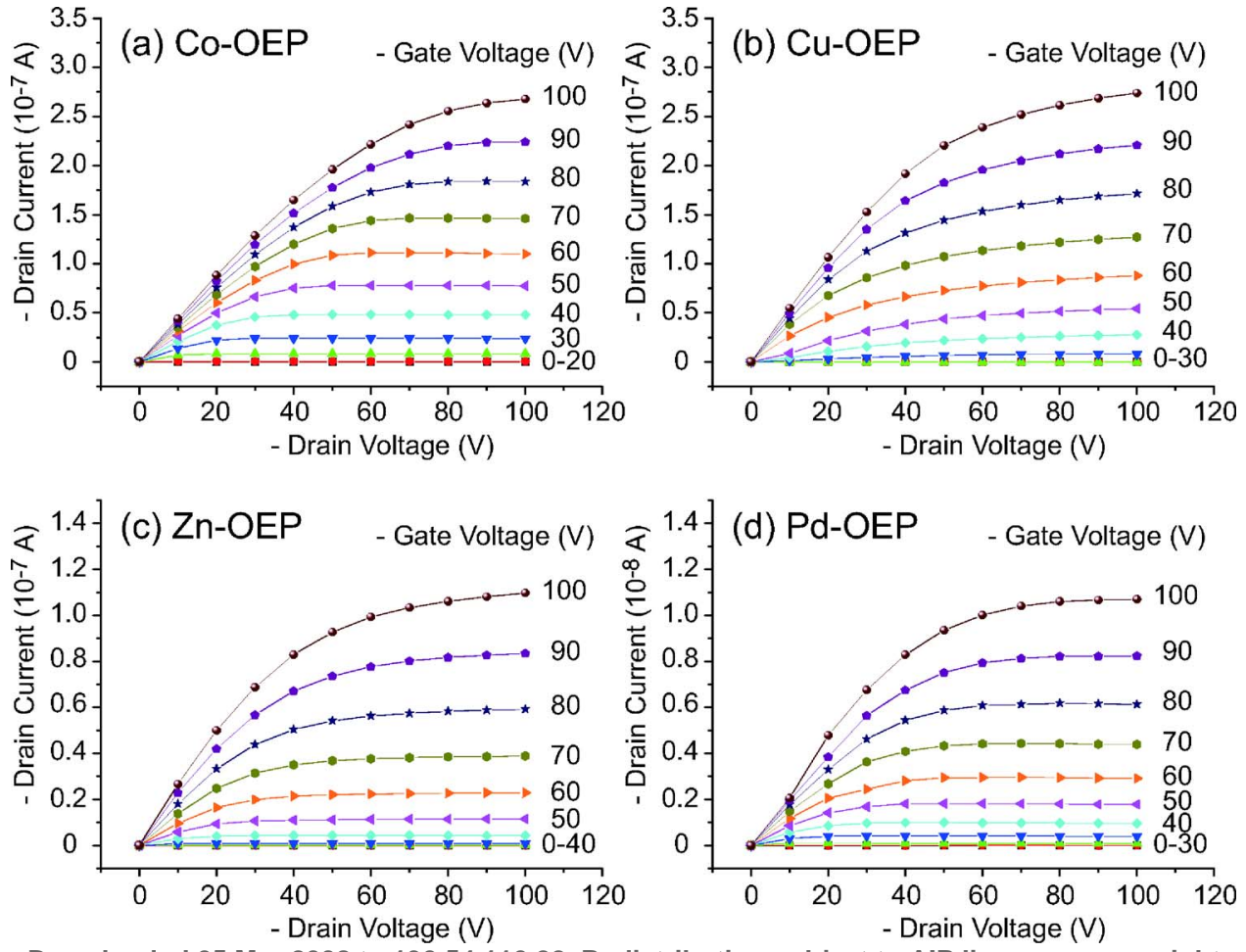

FIG. 2. (Color online) Current-voltage characteristics of OFETs based on M-OEP crystals. (a) Co-OEP with $W / L=0.08$, (b) $\mathrm{Cu}$-OEP with $W / L$ $=0.22$, (c) Zn-OEP with $W / L=0.20$, and (d) Pd-OEP with $W / L=0.036$. Gate voltage was swept from 0 to $-100 \mathrm{~V}$ in increments of $10 \mathrm{~V}$. 


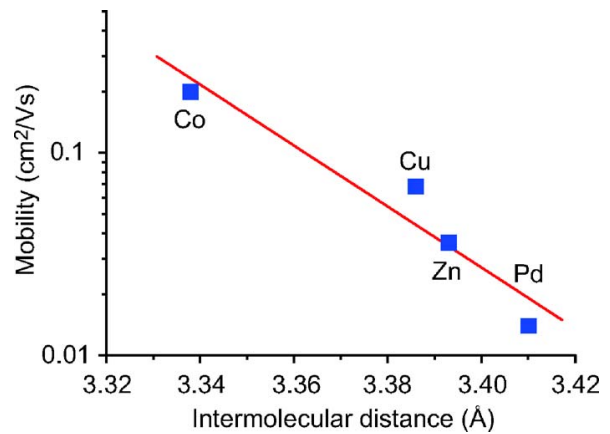

FIG. 3. (Color online) Dependence of $\mu_{\mathrm{FET}}$ on intermolecular distance in M-OEP crystals. Solid line is a guide for the eye.

present devices $\left(0.20 \mathrm{~cm}^{2} / \mathrm{V} \mathrm{s}\right)$, and all values are two to three orders of magnitude higher than those previously reported for M-OEP thin-film transistors. ${ }^{27}$ This can be attributed to the elimination of the grain boundary effect, indicating that these measurements reflect the intrinsic charge transport properties of the crystals.

The intermolecular distance among neighboring molecules was determined as the distance between the leastsquares plane of 25 atoms in a molecule and the center metal of the neighboring molecule [Fig. 1(b)]. The four M-OEP crystals exhibit different intermolecular distances. One origin of the difference would be the relatively large size of the center metal atoms; larger center atom tends to give increased intermolecular distance. The $\mu_{\mathrm{FET}}$ is plotted against the intermolecular distance in Fig. 3. The mobility clearly increases with decreasing intermolecular distance. However, the distance between center metals of neighboring molecules (given by the $c$-axis length) does not appear to be closely related to $\mu_{\mathrm{FET}}$, indicating that enhanced charge transport arises due to overlap of the highest occupied molecular orbitals distributed around the complex molecule. That is, the contribution of the $d$ orbitals of center metals to conduction is relatively small. Thus, closer packing of molecules, which increases the overlap of $\pi$ orbitals, is expected to result in higher conduction and, thus, higher field-effect mobility.

In conclusion, it was revealed that the field-effect mobility of organic field-effect transistors increases with decreasing intermolecular distance in the organic crystal. The enhancement of $\mu_{\mathrm{FET}}$ can be attributed to increased $\pi$ overlap among neighboring molecules and consequent promotion of the charge transport. This result, the obvious evidence of the relationship between device mobility and crystal structure in OFETs, indicates that optimization of the crystal structure may be an important approach to enhancing the field-effect mobility of organic materials.
This study was supported in part by Grants-in-Aid for Scientific Research (16GS50219, 17069004, and 18201028) from the Ministry of Education, Culture, Sports, Science and Technology of Japan.

${ }^{1}$ M. Pope and C. E. Swenberg, Electronic Processes in Organic Crystals and Polymer, 2nd ed. (Oxford University Press, New York, 1999).

${ }^{2}$ K. Waragai, H. Akimichi, S. Hotta, and H. Kano, Phys. Rev. B 52, 1786 (1995).

${ }^{3}$ Y. Y. Lin, D. J. Gundlach, and T. N. Jackson, Appl. Phys. Lett. 72, 1854 (1998).

${ }^{4}$ G. Horowitz, M. E. Hajlaoui, and R. Hajlaoui, J. Appl. Phys. 87, 4456 (2000).

${ }^{5}$ A. N. Aleshin, J. Y. Lee, S. W. Chu, J. S. Kim, and Y. W. Park, Appl. Phys. Lett. 84, 5383 (2004).

${ }^{6}$ C. R. Newman, R. J. Chesterfield, J. A. Merlo, and C. D. Frisbie, Appl. Phys. Lett. 85, 422 (2004).

${ }^{7}$ G. Horowitz and M. E. Hajlaoui, Synth. Met. 122, 185 (2001).

${ }^{8}$ I. Yagi, K. Tsukagoshi, and Y. Aoyagi, Appl. Phys. Lett. 86, 103502 (2005).

${ }^{9}$ P. Ostoja, P. Maccagnani, M. Gazzano, M. Cavallini, J. C. Kengne, R. Kshirsagar, F. Biscarini, M. Melucci, M. Zambianchi, and G. Barbarella, Synth. Met. 146, 243 (2004).

${ }^{10}$ F. Dinelli, M. Murgia, P. Levy, M. Cavallini, and F. Biscarini, Phys. Rev. Lett. 92, 116802 (2004).

${ }^{11}$ M. D. Curtis, J. Cao, and J. W. Kampf, J. Am. Chem. Soc. 126, 4318 (2004).

${ }^{12}$ H. Moon, R. Zeis, E.-J. Borkent, C. Besnard, A. J. Lovinger, T. Siegrist, C. Kloc, and Z. Bao, J. Am. Chem. Soc. 126, 15322 (2004).

${ }^{13}$ M. Cavallini, P. Stoliar, J.-F. Moulin, M. Surin, P. Leclere, R. Lazzaroni, D. W. Breiby, J. W. Andreasen, M. M. Nielsen, P. Sonar, A. C. Grimsdale, K. Mullen, and F. Biscarini, Nano Lett. 5, 2422 (2005).

${ }^{14}$ M. Ichikawa, H. Yanagi, Y. Shimizu, S. Hotta, N. Suganuma, T. Koyama, and Y. Taniguchi, Adv. Mater. (Weinheim, Ger.) 14, 1272 (2002).

${ }^{15}$ V. Podzorov, V. M. Pudalov, and M. E. Gershenson, Appl. Phys. Lett. 82, 1739 (2003).

${ }^{16}$ J. Takeya, C. Goldmann, S. Haas, K. P. Pernstich, B. Ketterer, and B. Batlogg, J. Appl. Phys. 94, 5800 (2003).

${ }^{17}$ R. W. I. de Boer, T. M. Klapwijk, and A. F. Morpurgo, Appl. Phys. Lett. 83, 4345 (2003).

${ }^{18}$ T. Minari, T. Nemoto, and S. Isoda, J. Appl. Phys. 96, 769 (2004).

${ }^{19}$ V. C. Sundar, J. Zaumseil, V. Podzorov, E. Menard, R. L. Willett, T. Someya, M. E. Gershenson, and J. A. Rogers, Science 303, 1644 (2004).

${ }^{20}$ J. Y. Lee, S. Roth, and Y. W. Park, Appl. Phys. Lett. 88, 252106 (2006).

${ }^{21}$ R. Zeis, T. Siegrist, and C. Kloc, Appl. Phys. Lett. 86, 022103 (2005).

${ }^{22}$ P. B. Shea, J. Kanicki, L. R. Pattison, P. Petroff, M. Kawano, H. Yamada, and N. Ono, J. Appl. Phys. 100, 034502 (2006).

${ }^{23}$ H. Wang, D. Song, J. Yang, B. Yu, Y. Geng, and D. Yan, Appl. Phys. Lett. 90, 253510 (2007).

${ }^{24}$ A. Ozarowski, H. M. Lee, and A. L. Balch, J. Am. Chem. Soc. 125, 12606 (2003).

${ }^{25}$ W. R. Scheidt and I. Turowska-Tyrk, Inorg. Chem. 33, 1314 (1994).

${ }^{26}$ R. Pak and W. R. Scheidt, Acta Crystallogr., Sect. C: Cryst. Struct. Commun. 47, 431 (1991).

${ }^{27}$ Y.-Y. Noh, J.-J. Kim, Y. Yoshida, and K. Yase, Adv. Mater. (Weinheim, Ger.) 15, 699 (2003). 